\title{
Combined effects of inversion and feature removal on N170 responses elicited by faces and car fronts
}

\author{
Nadine Kloth ${ }^{a, b, c,}{ }^{,}$, Roxane J. Itierd, and Stefan R. Schweinberger ${ }^{a, c}$ \\ aDepartment of Psychology, Friedrich Schiller University of Jena, Germany \\ ${ }^{\mathrm{b}} \mathrm{ARC}$ Centre of Excellence in Cognition and its Disorders, School of Psychology, The University \\ of Western Australia, Australia \\ 'DFG Research Unit Person Perception, Friedrich Schiller University of Jena, Germany \\ dDepartment of Psychology, University of Waterloo, Waterloo, Canada
}

\begin{abstract}
The face-sensitive N170 is typically enhanced for inverted compared to upright faces. Itier, Alain, Sedore, and McIntosh (2007) recently suggested that this N170 inversion effect is mainly driven by the eye region which becomes salient when the face configuration is disrupted. Here we tested whether similar effects could be observed with non-face objects that are structurally similar to faces in terms of possessing a homogeneous within-class first-order feature configuration. We presented upright and inverted pictures of intact car fronts, car fronts without lights, and isolated lights, in addition to analogous face conditions. Upright cars elicited substantial N170 responses of similar amplitude to those evoked by upright faces. In strong contrast to face conditions however, the car-elicited N170 was mainly driven by the global shape rather than the presence or absence of lights, and was dramatically reduced for isolated lights. Overall, our data confirm a differential influence of the eye region in upright and inverted faces. Results for car fronts do not suggest similar interactive encoding of eye-like features and configuration for non-face objects, even when these objects possess a similar feature configuration as faces.
\end{abstract}

\section{Keywords}

N170; Faces; Cars; Eyes; Inversion; Specificity

\section{Introduction}

One of the most important indicators of a qualitatively different processing of faces compared to other objects is the so-called face inversion effect. While upside-down presentation of objects generally interferes with their recognition, inversion of faces results in disproportional face perception and recognition impairments (Yin, 1969). This strong preference for upright presentation has generally been interpreted as indicating the great reliance of face perception mechanisms on configural information, which is known to be severely disrupted when faces are presented upside-down (Maurer, Le Grand, \& Mondloch, 2002; Rossion \& Gauthier, 2002).

(C) 2013 Elsevier Inc. All rights reserved.

Corresponding author at: School of Psychology (M304), The University of Western Australia, 35 Stirling Highway, Crawley, WA 6009, Australia. Fax: +61 86488 1006. nadine.kloth@uwa.edu.au (N. Kloth). 
The pronounced sensitivity of face processing mechanisms to stimulus orientation, as revealed by the inversion effect, is reflected in a strong sensitivity of the face-elicited N170 to inversion. The N170 is a negative-going event-related potential (ERP) recorded at occipitotemporal electrodes, which is typically observed when participants are presented with human faces (Bentin, McCarthy, Perez, Puce, \& Allison, 1996). The component is believed to reflect structural encoding, that is, the formation of a uniform representation of a seen face (Eimer, 1998, 2000; Rossion et al., 1999; Schweinberger \& Burton, 2003). The N170 is typically larger for faces than other visual stimuli, suggesting a large degree of facesensitivity (Itier \& Taylor, 2004; Rossion \& Jacques, 2008). Some object categories such as car fronts, however, can elicit large N170 responses (Rossion et al., 2000) that have sometimes been found to be indistinguishable from face-elicited responses in both amplitude and topography (Schweinberger, Huddy, \& Burton, 2004). Greebles, artificial objects with similar configural structure and intra-class homogeneity as faces, can also elicit substantial N170 responses after sufficient exposure, such that the N170 was sometimes taken to reflect visual expertise (Tanaka \& Curran, 2001). The sensitivity of the N170 to face orientation is typically revealed by a substantial increase in both amplitude and latency for inverted faces compared to upright faces (e.g., Bentin et al., 1996; Itier \& Taylor, 2004; Rossion et al., 2000), whereas inverting objects only results in a delayed latency (Itier, Latinus, \& Taylor, 2006). Although it is a well-established finding in ERP research on face processing, the mechanisms underlying the N170 inversion effect are still not well understood. The integration of behavioral and ERP findings suggests that the N170 reflects the structural encoding of a face, and likely its configural analysis which is known to be hampered for inverted faces. This impaired structural encoding might be reflected in the amplitude increase typically observed in the N170 to inverted faces, although it may seem counterintuitive that the disruption of a perceptual process is accompanied by an increase, rather than a decrease, in neural activation.

A recently proposed model offers a possible explanation for this apparent paradox. Itier and colleagues suggested that the N170 is potentially generated by the activity of separate groups of face-sensitive and eye-sensitive cells in occipitotemporal regions of the brain (Itier, Alain, Sedore, \& McIntosh, 2007; for an updated model see Itier \& Batty, 2009). As single-unit recordings in monkey temporal cortex have shown that eye-selective neurons do not respond when faces are presented upright (Perrett et al., 1985), the model assumes that the N170 to upright faces is mainly driven by "face cells" activity. However, when a face is inverted, the concomitant configural disruption is thought to disrupt the inhibitory effect of the face context on the "eye cells", so that in this case "eye cells" additionally contribute to the generation of the N170, resulting in a larger amplitude compared to upright face presentation.

Itier et al. (2007) tested their model's predictions by examining the N170 to presentations of upright and inverted faces, faces without eye region, and isolated eyes. They found a clear N170 inversion effect for intact faces but a strongly reduced inversion effect for faces without eye region. Irrespective of orientation, faces without eye region evoked N170 responses comparable in amplitude to those evoked by upright intact faces. Isolated eyes evoked large N170 amplitudes irrespective of their orientation, and of comparable magnitude to those evoked by inverted intact faces. This finding is well in line with the model which predicts a response from both the eye and face cells when isolated eyes are presented. Overall, these data supported the model's predictions in showing that it is the presence of eyes without an intact (i.e., upright) face context that leads to a dramatic increase in N170 amplitude (see also Eimer, Kiss, \& Nicholas, 2010; Nemrodov \& Itier, 2011). 
The purpose of the present study was to test the extent to which Itier et al.'s (2007) findings are specific to the eyes, which are usually considered the most important feature within the human face, and their interaction with the rest of the face. More specifically, we investigated whether the observed contribution of the presence or absence of the eye region to the N170 amplitude partly reflects a part-whole phenomenon which might be equally observable in non-face stimuli. On the one hand, as far as faces are concerned, there is some evidence for a special role of eyes compared to other features, such as the mouth. Eyes presented in isolation have been shown to elicit larger N170 than intact faces (Bentin et al., 1996; Itier, Van Roon, \& Alain, 2011; Itier et al., 2006, 2007; Taylor et al., 2001) while noses or mouths in isolation elicit object-like responses (Bentin et al., 1996) or delayed N170s (Nemrodov \& Itier, 2011). Moreover, mouthless faces, when presented upside-down, show similar inversion effects as intact faces (Nemrodov \& Itier, 2011), whereas eyeless faces do not, with either a complete lack of an inversion effect (Itier et al., 2011) or a significantly reduced one (Itier et al., 2007; Nemrodov \& Itier, 2011).

On the other hand, as mentioned above, several non-face categories such as car fronts elicit a large N170 and it is possible that the presence or absence of crucial parts of these stimuli might similarly impact the N170. A recent study showed that the presence or absence of the head in human body stimuli has similar effects on the N170 body inversion effect as the presence or absence of eyes on the face inversion effect (Mohamed, Neumann, \& Schweinberger, 2011). This study showed that the normal N170 body inversion effect (cf., Minnebusch, Suchan, \& Daum, 2009) was significantly reduced for bodies without heads. In analogy to Itier et al.'s (2007) assumptions about the relative contributions of "eye cells" and "face cells" to the N170 face inversion effect, Mohamed et al. (2011) suggested that the N170 body inversion effect could partly reflect the additional recruitment of head-sensitive cells which could be inhibited by an upright body context. Thus, a more general degree of hierarchical coding might be driving the influence of the presence and absence of face/body parts on the respective N170 face/body inversion effect. Importantly for present purposes, such hierarchical coding might not be specific to faces and body parts but might extend to other objects with homogeneous within-class first-order feature configurations.

In the present study, we compared the N170 evoked by car fronts vs. faces. We selected car fronts because they evoke a prominent N170 response (Schweinberger et al., 2004), and because they possess homogeneous within-class structural characteristics, and thus can be considered "face-like", at least with respect to these characteristics. Car fronts (i) are largely symmetric across the vertical axis, (ii) contain single features in the vertical midline, (iii) contain pairwise lateral features, and (iv) exhibit a largely uniform first-order spatial configuration of those features across exemplars. Finally, and of particular importance for the present study, headlights in cars may be considered as potential equivalents to eyes in faces.

We presented participants with pictures of intact faces, faces without eye region, and isolated eye regions as well as the analogous conditions of intact cars, cars without lights, and isolated lights in upright and inverted orientation. For faces, we expected to replicate the findings of Itier et al. (2007) of increased N170 amplitudes for inverted faces and a lack of such an inversion effect, or a reduced one, for faces without eye region and isolated eyes. To the extent that these N170 variations reflect a more general pattern of hierarchical coding in stimuli with homogeneous first-order configurations (Mohamed et al., 2011), a similar pattern of results would be predicted for faces and car fronts. Alternatively, if the pattern observed by Itier et al. (2007) is specific to the processing of faces and their most important feature, the eye region, one would expect a different contribution of car lights to the carelicited N170 response than the contribution of the eye region to the face-elicited N170 response. 


\section{Methods}

\subsection{Participants}

Sixteen undergraduate students from the University of Jena (6 male, 20-27 years, $M=22.8$ years $\pm 2.32 S D$ ) contributed data and received course credit or payment for their participation. All participants reported normal or corrected-to-normal vision and were righthanded according to a modified version of the Edinburgh Handedness Inventory (Oldfield, 1971). None of the participants reported a history of neurological or psychiatric disorders or received central-acting medication. The study was conducted in accordance with the ethical guidelines of the Declaration of Helsinki.

\subsection{Stimuli}

Grayscale photographs of 48 different human faces ( 24 female) and 48 car fronts were used to create the stimulus set. The face stimuli were taken from Itier et al. (2007). The intact version of these faces had been manipulated using Adobe Photoshop to create a "face without eye region", removing the eyes and eyebrows, and an "isolated eye region", consisting of only the eyes and eyebrows, for each individual (for details on the stimulus preparation, see Itier et al., 2007). For the purpose of the present study, equivalent manipulations were carried out on the pictures of 48 car fronts creating additional "cars without lights" and "isolated lights" stimuli in addition to the "intact" car stimuli (see Fig. 1 for stimulus examples).

Face and car stimuli were normalized according to their height. Face stimuli were about 7.5 $\mathrm{cm}$ high and varied in width from about 5-7 cm, depending on the proportions of the individual face. For the "isolated eye region" condition, faces were fitted behind a rectangular mask of $2.6 \mathrm{~cm}$ height and $1.6 \mathrm{~cm}$ width occluding the whole face apart from the eye region. Sizes of car stimuli were adjusted to the height of the face stimuli $(7.5 \mathrm{~cm})$ and varied in width from approximately $7.0 \mathrm{~cm}$ to $9.5 \mathrm{~cm}$ depending on the individual car's proportions. For the "isolated lights" condition, cars were fitted behind the same rectangular mask as used for the face stimuli. For this purpose, car stimuli had to be slightly downscaled so that both lights were visible within the mask (see Fig. 1). Finally, 43 grayscale pictures of butterflies were used. Using the SHINE tool-box (Willenbockel et al., 2010), all stimuli were equated for luminance and contrast.

\subsection{Design and procedure}

Stimulus Class (faces, car fronts), Presentation Condition (intact, stimulus w/o eye region/ lights, isolated eye region/lights), and Orientation (upright, inverted) were varied within participants, leading to 12 experimental conditions. Each condition comprised 48 different items/trial, resulting in a total of 576 trials which were presented in random order. To ensure that participants attentively fixated the presented stimuli, 43 butterflies were presented twice (86 presentations, corresponding to $\sim 15 \%$ of the trials), randomly intermixed, and participants were instructed to respond to these stimuli by pressing the space bar of a computer keyboard.

Within each trial, a stimulus was presented for $250 \mathrm{~ms}$ followed by a black fixation cross. The interstimulus interval randomly varied between $1000 \mathrm{~ms}$ and $1300 \mathrm{~ms}$ with a mean duration of $1150 \mathrm{~ms}$. A self-paced break was inserted after the first half of the trials. In total, the experiment lasted approximately $15 \mathrm{~min}$.

\subsection{Electrophysiologial recordings}

The EEG was recorded from $32 \mathrm{Ag} / \mathrm{AgCl}$ electrodes (AC, 0.05-40 Hz, $500 \mathrm{~Hz}$ sampling rate) at locations Fz, Cz, Pz, Iz, Fp1, Fp2, F3, F4, C3, C4, P3, P4, O1, O2, F7, F8, T7, T8, 
P7, P8, F9, F10, FT9, FT10, TP9, TP10, P9, P10, PO9, and PO10, with Cz as initial common reference, using an Easy-Cap ${ }^{\mathrm{TM}}$. Electrode impedances were kept below $10 \mathrm{k} \Omega$. The horizontal electro-oculogram (EOG) was recorded from the outer canthi of both eyes, and the vertical EOG was monitored bipolarly from above and below the right eye. Off-line, trials with non-ocular artifacts were discarded. For all other trials, ${ }^{1}$ the EEG was corrected for contributions of vertical and horizontal eye movements using BESA's automatic EOG artifact correction based on an adaptive artifact correction algorithm (Ille, Berg, \& Scherg, 2002). Data were segmented into trials of $600 \mathrm{~ms}$ (200 ms pre-stimulus baseline up to 400 $\mathrm{ms}$ after stimulus onset), digitally low-pass filtered at $40 \mathrm{~Hz}$ (zero phase shift, $12 \mathrm{~dB} / \mathrm{oct}$ ), and recalculated to average reference.

\subsection{ERP analyses}

For P100, peak amplitudes in the time segment between 80 and $120 \mathrm{~ms}$ post stimulus onset were analyzed at occipital electrode locations $\mathrm{O} 1$ and O2. For N170, peak amplitudes between 120 and $220 \mathrm{~ms}$ were analyzed at posterior electrodes P7 and P8 where the N170 was maximal. In two participants, N170 was lateralized to such an extreme degree that there was no obvious peak over the left or right hemisphere, respectively. For these participants, the amplitude for each condition was determined at the latency at which this condition peaked over the right or left hemisphere, respectively. ${ }^{2}$

ERP components were analyzed using repeated measures ANOVAs with Hemisphere (left, right), Stimulus Class (faces, car fronts), Presentation Condition (intact, stimulus w/o eye region/lights, isolated eye region/lights), and Orientation (upright, inverted) as within subject factors. Where appropriate, epsilon corrections for heterogeneity of covariances were performed (Huynh \& Feldt, 1976) and post hoc $t$ tests were corrected according to the Bonferroni-procedure $(a=.05)$.

\section{Results}

\subsection{P100}

The analysis of P100 peak amplitudes revealed a significant main effect of Orientation $\left(F[1,15]=4.72, p<.05, \eta_{p}^{2}=.24\right)$ and an interaction between Presentation Condition and Orientation $\left(F[2,30]=4.08, p<.05, \eta_{p}^{2}=.21\right)$, both qualified by a three-way interaction between Stimulus Class, Presentation Condition, and Orientation $(F[2,30]=3.66, p<.05$, $\left.\eta_{p}^{2}=.19\right)$. To follow up on the three-way interaction, we conducted separate analyses for faces and cars, considering Presentation Condition and Orientation as factors.

For faces, the ANOVA revealed a significant interaction of Presentation Condition and Orientation $\left(F[2,30]=4.04, p<.05, \eta_{p}^{2}=.21\right)$, reflecting a significant inversion effect for faces without eye region, with larger P100 amplitudes for inverted than upright presentation, $(t[15]=3.67, p<.01)$, but no inversion effects for intact faces $(t[15]=1.06, p=.31)$ or isolated eye regions $(t[15]=1.28, p=.22)$. Further, P100 amplitude was larger in response to isolated eye regions than intact faces in upright $(t[15]=2.71, p<.05)$ but not in inverted orientation $(t[15]=1.09, p=.29)$.

\footnotetext{
${ }^{1}$ The average number of trials per condition per subject included in the statistical analyses ranged from 30 to 48 trials $(M=43, S D=$ 3.9).

${ }^{2}$ To confirm that this procedure did not influence the results, identical analyses were performed with these two participants excluded. These analyses replicated the results reported here.
} 
An analogous ANOVA for car fronts only revealed a significant interaction of Presentation

Condition and Orientation $\left(F[2,30]=3.41, p<.05, \eta_{p}^{2}=.19\right)$, reflecting larger $\mathrm{P} 100$ amplitudes for inverted than upright intact cars only $(t[15]=2.39, p<.05)$.

\subsection{N170 amplitude}

N170 peak amplitude analyses ${ }^{3}$ revealed a significant main effect of Stimulus Class $(F[1,15]$ $=23.70, p<.001, \eta_{p}^{2}=.61$ ), and significant two-way interactions of Presentation Condition with Orientation $\left(F[2,30]=9.88, p=.001, \eta_{p}^{2}=.40\right)$ and Presentation Condition with Stimulus Class $\left(F[2,30]=7.07, p=.003, \eta_{p}^{2}=.32\right)$. These effects were qualified by a prominent three-way interaction between Stimulus Class, Presentation Condition, and Orientation $\left(F[2,30]=12.95, p<.001, \eta_{p}^{2}=.46\right)$. To follow up on this interaction, we performed separate analyses for faces and car fronts, with Presentation Condition and Orientation as factors.

For faces, a significant interaction of Presentation Condition and Orientation $(F[2,30]=$ $\left.17.02, p<.001, \eta_{p}^{2}=.53\right)$ was found. As predicted, planned comparisons revealed significant N170 inversion effects with larger amplitudes for inverted than upright intact faces $(t[15]=$ $3.64, p=.002)$, but no inversion effects for faces without eye region $(t[15]=0.25, p=.81)$ or isolated eye regions $(t[15]=1.58, p=.135)$, see Fig. 2 . Further, for upright face stimuli, N170 amplitudes were larger for isolated eye regions compared to both intact faces $(t[15]=$ $2.92, p=.011)$ and faces without eye region $(t[15]=2.25, p=.04)$, which did not differ from each other $(t[15]=1.19, p=.25)$. For inverted face stimuli, intact faces evoked larger N170 amplitudes than isolated eye regions $(t[15]=2.58, p=.021)$ and faces without eye region $(t[15]=3.81, p=.002)$, which did not differ from each other $(t[15]=0.68, p=.51)$.

For car fronts, the ANOVA revealed a main effect of Presentation Condition $(F[2,30]=$ $\left.14.80, p<.001, \eta_{p}^{2}=.50\right)$. Irrespective of orientation, isolated lights evoked smaller amplitudes than both intact cars $(t[15]=4.52, p<.001$ and $t[15]=4.54, p<.001$, for upright and inverted conditions, respectively) and cars without lights $(t[15]=2.52, p=.023$ and $t[15]=3.99, p=.001$, for upright and inverted conditions, respectively), see Figs. 3 and 4.

Looking into the question of face-specificity of the N170, we compared responses to faces vs. cars in the different experimental conditions. Remarkably, the difference in N170 amplitude evoked by upright intact faces vs. upright intact cars failed to reach significance $(t[15]=1.42, p=.18)$ whereas face stimuli reliably evoked larger N170 amplitudes than car stimuli in all other experimental conditions (all $t \mathrm{~s}[15]>2.70$, all $p \mathrm{~s}<.02$ ), although this difference failed to survive Bonferroni-correction for stimuli without eye regions/lights in both upright $\left(t[15]=2.75, p_{\text {uncorrected }}=.015\right)$ and inverted conditions $(t[15]=2.79$, $\left.p_{\text {uncorrected }}=.014\right)$, see Fig. 5 .

\section{3. $\mathrm{N} 170$ latency}

An equivalent ANOVA on N170 peak latencies revealed significant main effects of Stimulus Class $\left(F[1,15]=20.60, p<.001, \eta_{p}^{2}=.58\right)$, Presentation Condition $(F[2,30]=$ $\left.41.09, p<.001, \eta_{p}^{2}=.73\right)$, and Orientation $\left(F[1,15]=70.46, p<.001, \eta_{p}^{2}=.82\right)$, as well as a number of significant two-way interactions which were qualified by a significant three-way interaction of Stimulus Class, Presentation Condition, and Orientation $(F[2,30]=3.45, p=$.

\footnotetext{
${ }^{3}$ Additional analyses, based on mean amplitudes in a time window of $\pm 20 \mathrm{~ms}$ around the peak latency of each condition, fully replicated the findings reported here. These additional analyses are therefore not reported to avoid redundancy.
} 
$044, \eta_{p}^{2}=.19$ ). Again, separate analyses for faces and car fronts were performed to follow up on the three-way interaction.

For face stimuli, the ANOVA revealed main effects of Presentation Condition $(F[2,30]=$ $\left.29.77, p<.001, \eta_{p}^{2}=.67\right)$ and Orientation $\left(F[1,15]=30.36, p<.001, \eta_{p}^{2}=.67\right)$ and an interaction of these factors $\left(F[2,30]=6.98, p=.014, \eta_{p}^{2}=.32\right)$. Planned comparisons revealed that inversion substantially delayed N170 latencies for intact faces $(t[15]=-11.23$, $p<.001)$ but less so for faces without eye region $(t[15]=-2.13, p=.05)$ and isolated eye regions $(t[15]=-2.75, p=.02)$. For upright and inverted face stimuli, N170 latencies evoked by intact stimuli were significantly shorter than those evoked by faces without eye region $(t[15]=-4.77, p<.001, t[15]=-5.65, p<.001$, for upright and inverted conditions, respectively) and isolated eye regions $(t[15]=-7.55, p<.001$ and $t[15]=5.98, p<.001$, for upright and inverted conditions, respectively), which did not differ significantly (both $t[15] \mathrm{s}$ $<1$, both $p$ s $>.50$ ).

For car stimuli, the ANOVA revealed significant main effects of Presentation Condition $\left(F[2,30]=36.37, p<.001, \eta_{p}^{2}=.71\right)$ and Orientation $\left(F[1,15]=20.15, p<.001, \eta_{p}^{2}=.57\right)$ as well as an interaction of these factors $\left(F[2,30]=3.48, p=.044, \eta_{p}^{2}=.19\right)$. Inversion significantly delayed the $\mathrm{N} 170$ in response to intact car fronts $(t[15]=5.26, p<.001)$ and cars without lights $(t[15]=4.49, p<.001)$ whereas latencies did not differ between upright and inverted presentation of isolated lights $(p>.20)$. Irrespective of orientation, isolated lights evoked delayed N170 responses compared to both intact car fronts $(t[15]=6.10, p<$. 001 and $t[15]=5.36, p<.001$, for upright and inverted conditions, respectively) and car fronts without lights $(t[15]=6.20, p<.001$ and $t[15]=4.83, p<.001$, for upright and inverted conditions, respectively, Fig. 4). Finally, we compared N170 latencies to faces vs. cars in each experimental condition. Irrespective of orientation, N170 latencies in response to the car conditions were significantly delayed relative to faces in the intact and the eyes/ lights only conditions (all $t \mathrm{~s}[15]>3.00$, all $p \mathrm{~s}<.01$ ) but not in the conditions without eyes/ lights (both $t[15] \mathrm{s}<1, p s>.30$ ), see Fig. 5 .

\section{Discussion}

The present study was designed to replicate and extend earlier research on the contribution of the eye region to the face-sensitive N170 component and the N170 face inversion effect. Typically, the N170 amplitude is enlarged and delayed in response to inverted as compared to upright faces (e.g., Bentin et al., 1996; Itier \& Taylor, 2004; Rossion et al., 2000). Itier et al. (2007) have proposed that this N170 inversion effect is largely driven by information in the eye region presented within an intact face. They suggested that both eye-sensitive and face-sensitive cells potentially contribute to the generation of the N170 component, but that eye-sensitive cells are inhibited when the eyes are presented within an upright face context, while both cell populations respond to isolated eyes and inverted face presentations. Consistent with this idea, Itier et al. (2007) showed that only intact faces evoked a much larger N170 amplitude when inverted, presumably due to the recruitment of both "face cells" and "eye cells". Faces without eyes, in contrast, were either found to produce a much smaller inversion effect (Itier et al., 2007; Nemrodov \& Itier, 2011) or no inversion effect at all (Itier et al., 2011) and isolated eye regions did not yield any inversion effect (Itier et al., 2006, 2007, 2011; Nemrodov \& Itier, 2011). Moreover, N170 amplitude in response to upright eye-less faces was equivalent to the amplitude evoked by upright intact faces whereas the amplitude in response to isolated eye regions (in both orientations) was equivalent to the one evoked by inverted intact faces. Intriguingly, a recent study observed a similar lack of inversion effects for bodies without heads as well as for faces without eyes 
(Mohamed et al., 2011). While these effects may reflect processes specific to visual person perception, they might also reflect a more general pattern of hierarchical coding of stimuli with homogenous within-class first-order feature configurations.

To follow up on this earlier work and clarify whether the contributions of crucial parts to N170 generation are specific to faces (and human bodies) or reflect a more general effect of hierarchical coding that might also be of importance for the processing of nonface objects, we examined whether or not a similar contribution of the presence or absence of headlights would be found in response to images of car fronts, a non-face stimulus class that is structurally similar to faces in terms of a number of characteristics, as detailed previously. The present N170 results for the face conditions largely replicated the findings by Itier et al. (2007), and the lack of equivalent effects in the P100 confirms that these mechanisms are indeed taking place in the N170 time window and are not just carried over from earlier components. Most importantly, N170 inversion effects were only observed for intact faces, whereas neither faces without eyes nor isolated eye regions evoked larger N170 amplitudes when inverted. Further in line with Itier et al.'s model (2007), N170 for upright stimuli was larger for isolated eye regions than both intact faces and faces without eye regions.

However, in contrast with the models assumption that eye cells and face cells respond equally to inverted intact faces and upright and inverted isolated eyes, inverted intact faces evoked even larger amplitudes than inverted isolated eyes. These findings could be tentatively related to a recent study by Eimer et al. (2010), which tested the predictions of Itier et al.'s (2007) model by means of rapid visual adaptation. Eimer et al. inferred relative contributions of eye-sensitive and face-sensitive cells to the N170 response from adaptation patterns of N170 amplitude in different adaptor- and test-stimulus pairings. Specifically, their finding of no measureable N170 adaptation effects in trials in which inverted faces were preceded by isolated eyes adaptors could suggest that the N170 to inverted intact faces originates from different neural generators than the N170 to isolated eyes (but see Nemrodov \& Itier, 2011).

The present results on N170 latencies to faces may reflect a sensitivity to the "faceness" (whereas N170 amplitude may be largely driven by the relative contributions of eye cells and face cells as proposed by Itier et al.'s model). For both upright and inverted faces, N170 latencies evoked by intact stimuli were significantly shorter than those evoked by faces without eyes (see Eimer, 1998; Itier et al., 2007, 2011 for similar findings) and isolated eyes (cf. Itier et al., 2006, 2007, 2011; Nemrodov \& Itier, 2011), with no difference between these two "incomplete" stimulus categories. In line with this, inversion effects on N170 latency were also most pronounced for intact faces and were reduced for faces without eye region and for isolated eye regions.

Replicating earlier research, faces and car fronts both evoked a substantial N170 in all three conditions. Overall, the N170 amplitude was more pronounced for faces than for car fronts, with the notable exception that this difference failed to reach statistical significance for the upright intact stimuli (for similar findings, see Schweinberger et al., 2004). With respect to N170 latencies, we observed delayed responses to cars compared to faces both when stimuli were intact and when eye regions/headlights were presented in isolation. However, there was no difference in N170 latencies between stimulus classes for faces and cars presented without eye regions and headlights, respectively. A similar pattern has earlier been reported for N170 latencies evoked by human and animal faces (Itier et al., 2011). Whereas N170 latencies were generally delayed in response to faces of non-human species compared to human faces, the latency difference was absent when faces were presented without eyes. Itier et al. (2011) suggested that the presence of human eyes might drive the temporal processing advantage for human faces over those of animals. The present data further 
support this idea and extend it to the comparison of brain responses evoked by faces and non-animate objects with homogeneous first-order configuration.

Crucially, strong qualitative differences between faces and car fronts were observed regarding the pattern of N170 modulation to both inversion and feature deletion, underlining the face sensitivity of these N170 effects. Although car fronts elicited a prominent N170, its amplitude was insensitive to inversion, replicating earlier findings indicating that inverted objects do not evoke enhanced N170 amplitudes (Eimer et al., 2010; Itier et al., 2006; Rossion et al., 2000, see also Itier et al., 2011, for similar findings for N170 amplitudes elicited by animal faces). Irrespective of stimulus orientation, intact cars and cars without lights consistently evoked larger and earlier N170 amplitudes than isolated lights. This pattern suggests that the car-elicited N170 is more sensitive to the overall outline of the car stimuli than to the presence of lights. This idea receives further support from the fact that the inversion effect in N170 latencies for cars was also restricted to those conditions in which the overall shape of the car was present, i.e., intact cars and cars without lights, which elicited delayed N170 peaks when presented inverted rather than upright.

The pattern of reduced N170 amplitudes to isolated headlights of cars is the exact opposite of what is typically observed for faces, with larger N170 responses to isolated eye regions compared to intact faces and faces without eyes. Thus, although the N170 to upright car fronts may respond to their overall configuration (albeit with a delay), the N170 does not respond to lights presented in isolation the way it responds to isolated eye regions. On the contrary, while lights evoke classic object-like N170 responses, human eyes evoke an even larger N170 than faces. Overall, and despite reports of similar N170 responses to upright intact faces and car fronts (Dering, Martin, Moro, Pegna, \& Thierry, 2011; Schweinberger et al., 2004), our results therefore confirm the face sensitivity of the N170, and underline the specific modulation by inversion and feature deletion as an important aspect of the N170 face sensitivity.

Our findings may also be considered in the context of recent claims of an anthropomorphism of car fronts, based on eye movement patterns which suggested an equivalent preference for looking at headlights in car fronts as found for eyes in faces (Windhager et al., 2010). Against those findings, the present results could suggest that lights in isolation lose their eye-likeness, and therefore this anthropomorphism, which would explain the present reduced and delayed N170 responses to isolated lights.

In line with other recent findings (Itier et al., 2011), we did not find any statistical evidence for an inversion effect in N170 amplitudes for faces without eye region. The original Itier et al. (2007) study, however, as well as a recent study by Nemrodov and Itier (2011) did report a significant inversion effect in this condition but of greatly reduced magnitude compared to intact faces. Visual inspection of Fig. 2 of the present article might similarly suggest a small increase of N170 amplitude in response to inverted compared to upright faces without eye region, at least at electrode $\mathrm{P} 8$. This effect might indicate that other mechanisms contribute to the prominent inversion effect observed in $\mathrm{N} 170$ amplitude, in addition to eye-sensitive and face-sensitive neural activity.

One potential (additional) contribution to the N170 inversion effect might be the recruitment of object-sensitive neurons whenever faces are inverted (Rossion et al., 2000). This suggestion was originally based on fMRI evidence showing that inverted faces activate regions typically engaged in object processing as well as those traditionally responding to upright faces (e.g., Haxby et al., 1999), and has recently received further support from intracranial recordings (Rosburg et al., 2010). Rosburg et al. (2010) compared responses at intracranial electrodes in lateral occipito-temporal regions when presenting either faces or 
objects (houses) in upright and inverted orientation. They found that electrodes which showed preferential responses to house over face stimuli displayed larger responses to inverted than upright faces as well as an equivalent inversion effect for houses. Electrodes that responded more strongly to faces than houses, however, only showed an inversion effect for faces and showed no orientationsensitivity for houses.

This intriguing evidence is well in line with behavioral findings suggesting that the visual system may treat inverted faces in a more object-like manner than upright faces. Future studies will have to explore these effects in more detail to ensure a better understanding of the relative contributions of face cells, eye cells, object-specific neurons, and potentially also orientations-sensitive neurons (see Eimer et al., 2010; Rhodes et al., 2004) to the N170 response to inverted faces.

The present results complement the findings of another recent study investigating the species-specificity of the contribution of the eye region to the N170 response elicited by upright and inverted faces (Itier et al., 2011). Although N170 amplitudes evoked by upright animal faces were even larger than those evoked by human faces, N170 amplitude was not enhanced in response to inverted animal faces (see also Wiese, Stahl, \& Schweinberger, 2009). An interesting difference between non-human faces and cars, however, is the fact that the car-elicited N170 amplitude observed in the present study seemed to be mainly driven by the presence of an intact car shape. This is in some contrast to the perception of non-human faces, for which N170 responses to eyeless faces were reduced in amplitude compared to intact faces, indicating that the presence of the eyes in these faces might be a necessary prerequisite for a maximal N170 amplitude. Together, these data suggest qualitative differences between N170s evoked by human faces, animal faces, and car fronts, in the sense that the orientation-sensitivity of N170 amplitude might be specific to human faces, whereas the presence of the eye region seems to play a more general role for animal faces. For cars, an object class with a special homogeneous within-class configuration, however, the presence of the overall shape seems to be the main factor eliciting an N170 response.

In conclusion, the present study provides important and novel information with respect to the combined effects of inversion and feature removal on the N170 ERP response. A comparison of effects elicited by faces and car fronts (here considered as non-face objects with a homogeneous within-class first-order configuration) revealed that, even though remarkably similar N170 responses were elicited by upright intact faces and car fronts, the N170 was delayed for car fronts and exhibited a response pattern to inversion and feature removal that was unique to human faces. These findings are generally in line with the model by Itier et al. (2007), which specifies interactions in the encoding of the eyes and the rest of the face in face perception. Importantly, findings for car fronts do not suggest that similar interactive encoding takes place for non-face objects, even when they are characterized by a homogenous configuration that might in some respects be considered face-like.

\section{Acknowledgments}

The authors thank Jeff Bruce, Bettina Kamchen, Andrea Kowallik, Katharina Merhof, Maria Rohmann, and Sina Schneider for their help in stimulus editing and data acquisition and Markus Neumann for his help with data analysis. This research was supported by the Australian Research Council Centre of Excellence in Cognition and its Disorders (Project Number CE110001021) and by grants from the Canada Institutes for Health Research (CIHR, MOP-89822) to RI and from the Deutsche Forschungsgemeinschaft to SRS (DFG Grant FOR 1097, Person Perception).

\section{References}

Bentin S, McCarthy G, Perez E, Puce A, Allison T. Electrophysiological studies of face perception in humans. Journal of Cognitive Neuroscience. 1996; 8:551-565. [PubMed: 20740065] 
Dering B, Martin CD, Moro S, Pegna AJ, Thierry G. Face-sensitive processes one hundred milliseconds after picture onset. Frontiers in Human Neuroscience. 2011; 5:93. http://dx.doi.org/ 10.3389/fnhum.2011.00093. [PubMed: 21954382]

Eimer M. Does the face-specific N170 component reflect the activity of a specialized eye processor? NeuroReport. 1998; 9:2945-2948. [PubMed: 9804295]

Eimer M. The face-specific N170 component reflects late stages in the structural encoding of faces. NeuroReport. 2000; 11:2319-2324. [PubMed: 10923693]

Eimer M, Kiss M, Nicholas S. Response profile of the face-sensitive N170 component: A rapid adaptation study. Cerebral Cortex. 2010; 20:2442-2452. [PubMed: 20080930]

Haxby JV, Ungerleider LG, Clark VP, Schouten JL, Hoffman EA, Martin A. The effect of face inversion on activity in human neural systems for face and object perception. Neuron. 1999; 22(1): 189-199. [PubMed: 10027301]

Huynh H, Feldt LS. Estimation of the Box correction for degrees of freedom from sample data in randomized block and split-plot designs. Journal of Educational Statistics. 1976; 1:69-82.

Ille N, Berg P, Scherg M. Artifact correction of the ongoing EEG using spatial filters based on artifact and brain signal topographies. Journal of Clinical Neurophysiology. 2002; 19:113-124. [PubMed: 11997722]

Itier RJ, Alain C, Sedore K, McIntosh AR. Early face processing specificity: It's in the eyes! Journal of Cognitive Neuroscience. 2007; 19(11):1815-1826. [PubMed: 17958484]

Itier RJ, Batty M. Neural bases of eye and gaze processing: The core of social cognition. Neuroscience and Biobehavioral Reviews. 2009; 33:843-863. [PubMed: 19428496]

Itier RJ, Latinus M, Taylor MJ. Face, eye and object early processing: What is the face specificity? NeuroImage. 2006; 29:667-676. [PubMed: 16169749]

Itier RJ, Taylor MJ. N170 or N1? Spatiotemporal differences between object and face processing using ERPs. Cerebral Cortex. 2004; 14:132-142. [PubMed: 14704210]

Itier RJ, Van Roon P, Alain C. Species sensitivity of early face and eye processing. NeuroImage. 2011; 54:705-713. [PubMed: 20650321]

Maurer D, Le Grand R, Mondloch CJ. The many faces of configural processing. Trends in Cognitive Sciences. 2002; 6:255-260. [PubMed: 12039607]

Minnebusch DA, Suchan B, Daum I. Losing your head: Behavioral and electrophysiological effects of body inversion. Journal of Cognitive Neuroscience. 2009; 21:865-874. [PubMed: 18702581]

Mohamed TN, Neumann MF, Schweinberger SR. Combined effects of attention and inversion on event related potentials to human bodies and faces. Cognitive Neuroscience. 2011; 2:138-146. [PubMed: 24168528]

Nemrodov D, Itier RJ. The role of eyes in early face processing: A rapid adaptation study of the inversion effect. British Journal of Psychology. 2011; 102:783-798. [PubMed: 21988384]

Oldfield RC. Assessment and analysis of handedness - Edinburgh Inventory. Neuropsychologia. 1971; 9(1):97-113. [PubMed: 5146491]

Perrett DI, Smith PAJ, Potter DD, Mistlin AJ, Head AS, Milner AD, et al. Visual cells in the temporal cortex sensitive to face view and gaze direction. Proceedings of the Royal Society of London, Series B: Biological Sciences. 1985; 223:293-317.

Rhodes G, Jeffery L, Watson TL, Jaquet E, Winkler C, Crawford CWG. Orientation-contingent face aftereffects and implications for face-coding mechanisms. Current Biology. 2004; 14:2119-2123. [PubMed: 15589154]

Rosburg T, Ludowig E, Dumpelmann M, Alba-Ferrara L, Urbach H, Elger CE. The effect of face inversion on intracranial and scalp recordings of event-related potentials. Psychophysiology. 2010; 47(1):147-157. [PubMed: 19761525]

Rossion B, Delvenne JF, Debatisse D, Goffaux V, Bruyer R, Crommelinck M, et al. Spatio-temporal localization of the face inversion effect: An event-related potentials study. Biological Psychology. 1999; 50:173-189. [PubMed: 10461804]

Rossion B, Gauthier I. How does the brain process upright and inverted faces? Behavioural and Cognitive Neuroscience Reviews. 2002; 1:62-74. 
Rossion B, Gauthier I, Tarr MJ, Despland P, Bruyer R, Linotte S, et al. The N170 occipito-temporal component is delayed and enhanced to inverted faces but not to inverted objects: An electrophysiological account of face-specific processes in the human brain. NeuroReport. 2000; 11:69-74. [PubMed: 10683832]

Rossion B, Jacques C. Does physical interstimulus variance account for early electrophysiological face sensitive responses in the human brain? Ten lessons on the N170. NeuroImage. 2008; 39:19591979. [PubMed: 18055223]

Schweinberger SR, Burton AM. Covert recognition and the neural system for face processing. Cortex. 2003; 39:9-30. [PubMed: 12627750]

Schweinberger SR, Huddy V, Burton AM. N250r: A face-selective brain response to stimulus repetitions. NeuroReport. 2004; 15:1501-1505. [PubMed: 15194883]

Tanaka JW, Curran T. A neural basis for expert object recognition. Psychological Science. 2001; 12:43-47. [PubMed: 11294227]

Taylor MJ, Edmonds GE, McCarthy G, Truett A. Eyes first! Eye processing develops before face processing in children. NeuroReport. 2001; 12(8):1671-1676. [PubMed: 11409737]

Wiese H, Stahl J, Schweinberger SR. Configural processing of other-race faces is delayed but not decreased. Biological Psychology. 2009; 81(2):103-109. [PubMed: 19428974]

Willenbockel V, Sadr J, Fiset D, Horne G, Gosselin F, Tanaka JW. Controlling low-level image properties: The SHINE toolbox. Behavior Research Methods. 2010; 42:671-684. [PubMed: 20805589]

Windhager S, Hutzler F, Carbon CC, Oberzaucher E, Schaefer K, Thorstensen T, et al. Laying eyes on headlights: Eye movements suggest facial features in cars. Collegium Antropologicum. 2010; 34(3):1075-1080. [PubMed: 20977106]

Yin R. Looking at upside-down faces. Journal of Experimental Psychology. 1969; 81:141-145. 

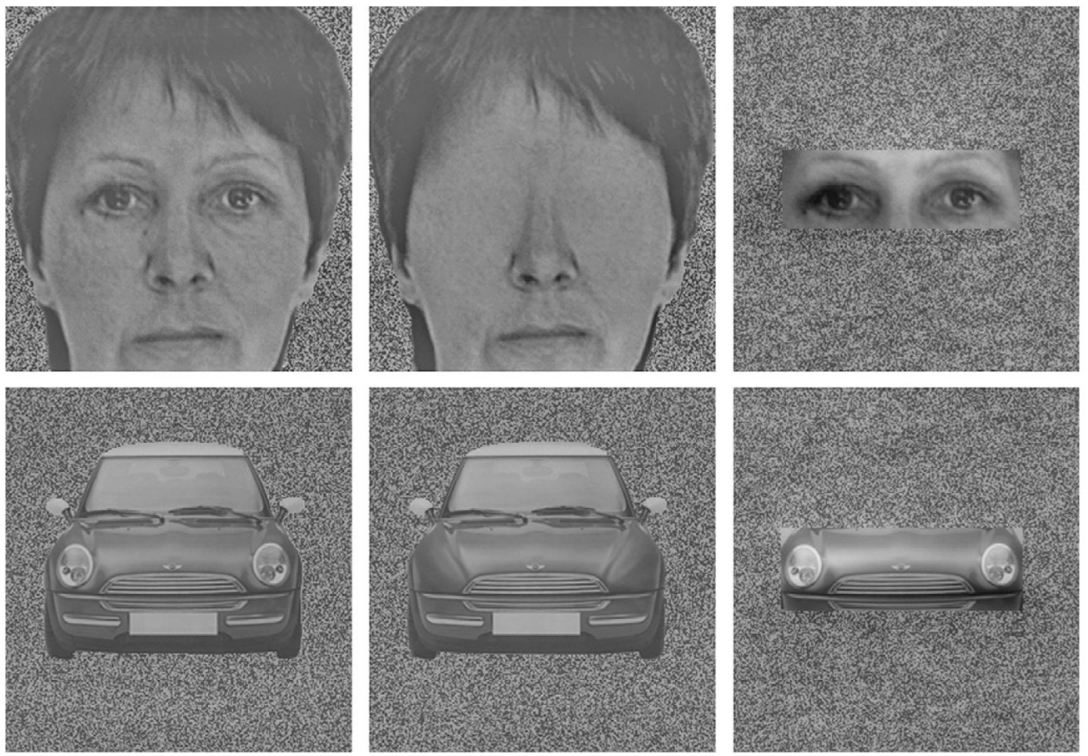

Fig. 1.

Stimulus examples. Top (left to right): intact face, face without eye region and isolated eye region. Bottom (left to right): intact car front, car without lights, and isolated lights. 

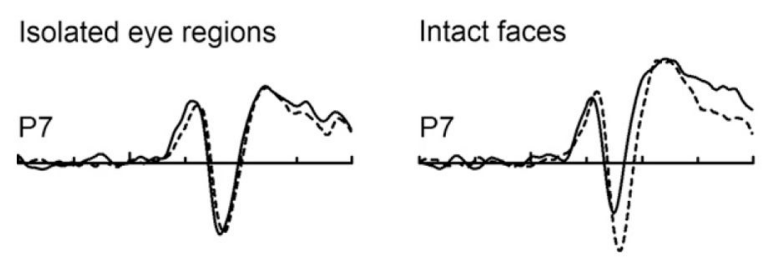

Faces without eye region
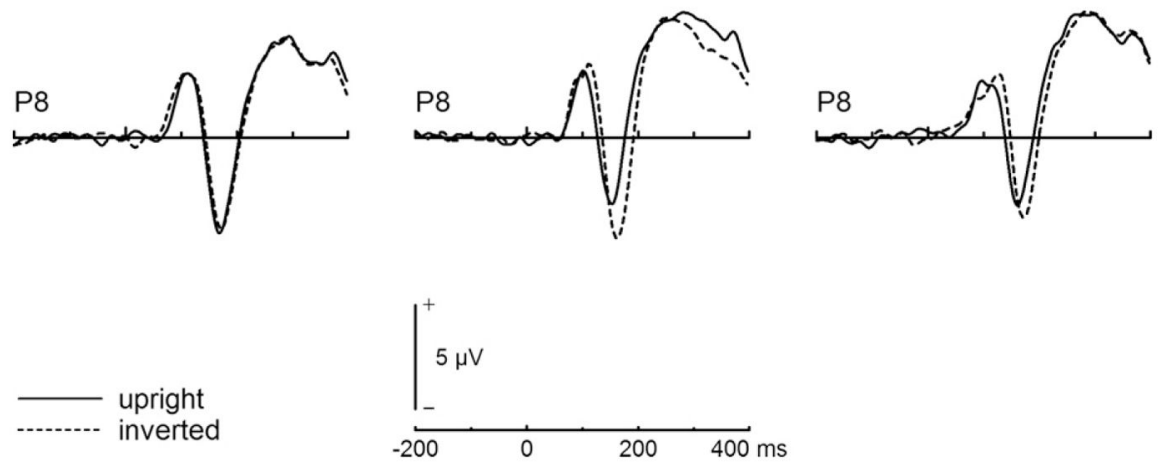

Fig. 2.

N170 inversion effects at electrodes P7 and P8 as observed in the three different face conditions. 

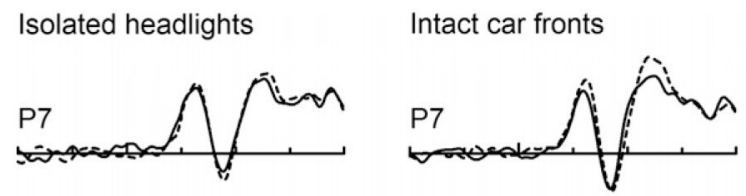

Car fronts without headlights
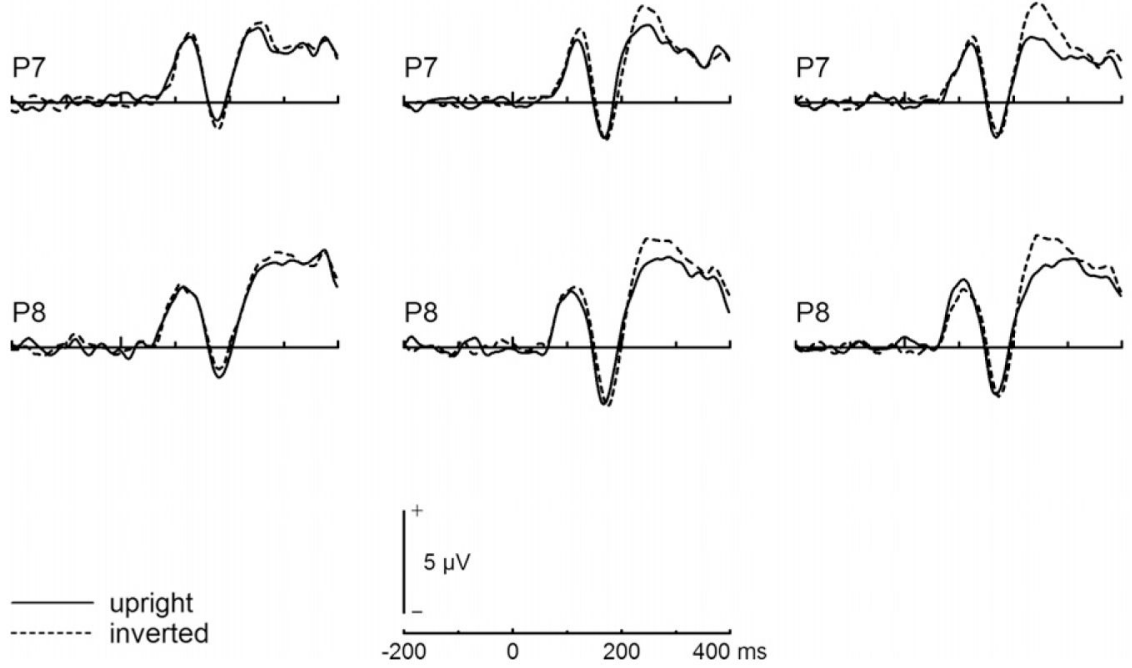

Fig. 3.

N170 inversion effects at electrodes P7 and P8 as observed in the three different car conditions. 


\section{Car conditions}
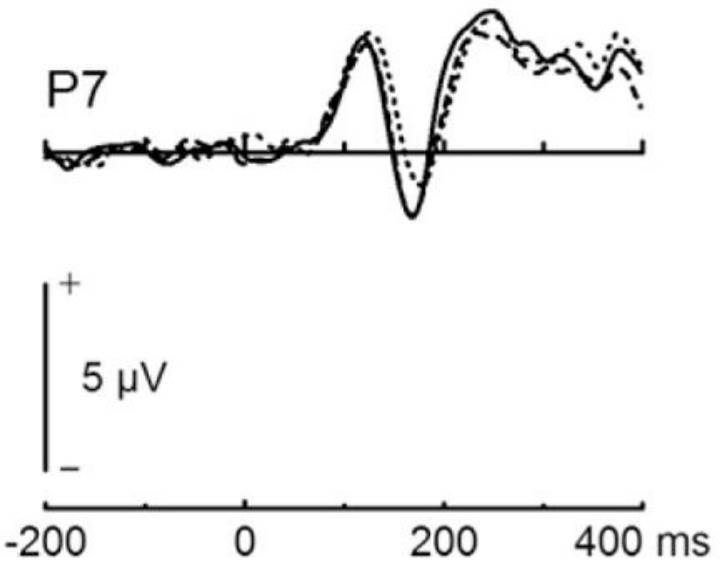
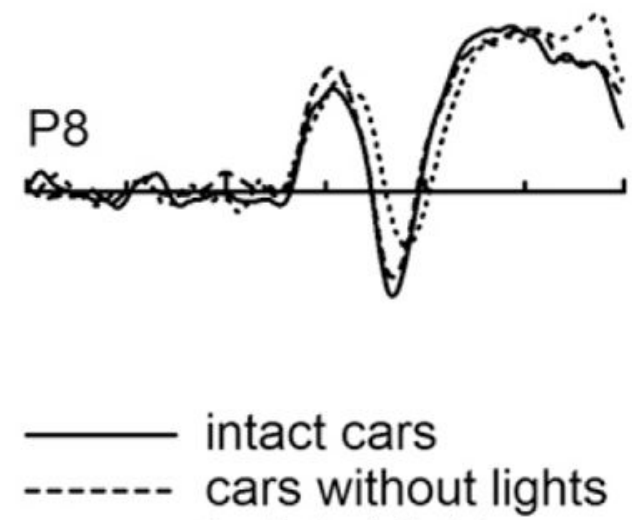
isolated lights

Fig. 4.

Effects of Presentation Condition (intact cars, cars without lights, isolated lights) on the N170 elicited by upright car stimuli. 
(a) Upright presentation

Intact stimuli

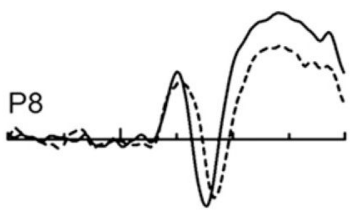

(b) Inverted presentation

Intact stimuli
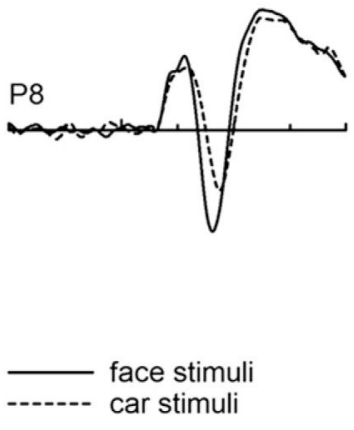

Without eye region/lights

Isolated eye regions/lights

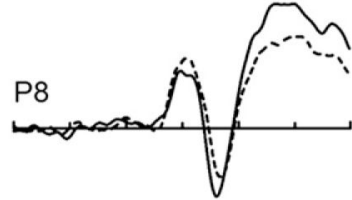

Without eye region/lights

Isolated eye regions/lights
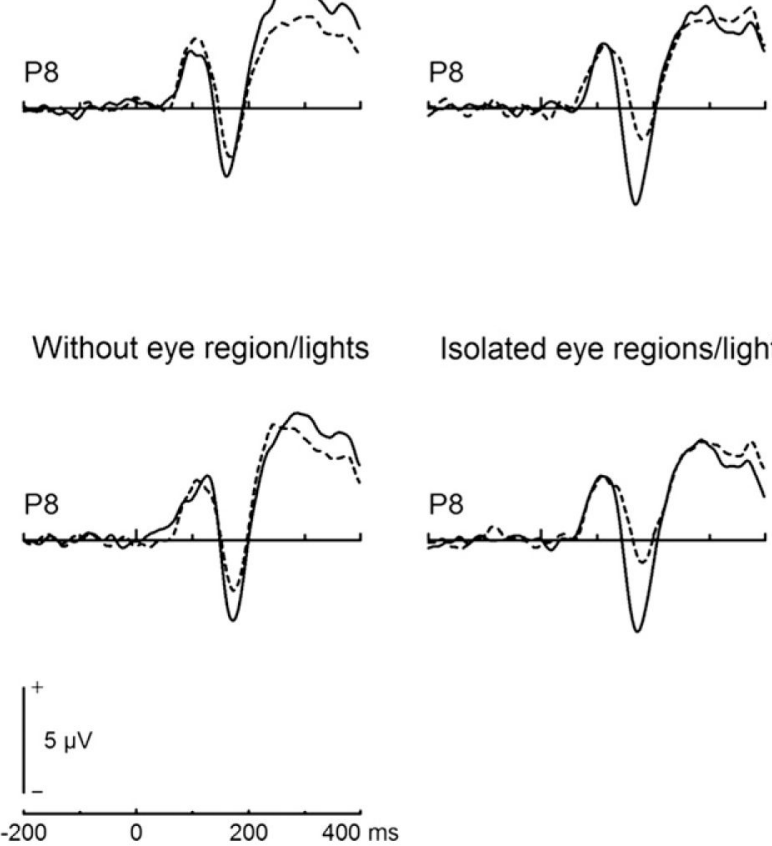

Fig. 5.

Comparison of N170 amplitudes and latencies elicited by faces and car fronts in each of the six different presentation conditions. 